and by the lemma and (18) for sufficiently large $n$

$$
\begin{aligned}
\Delta & \leqq \frac{\epsilon}{2 c_{1}} c_{1}+\sum_{1 \leqq k \leqq n,\left|\theta-\theta_{k}^{(n)}\right|>\delta} \frac{1}{2} 2 M\left|l_{k}[\theta-\pi / 2 n]+l_{k}[\theta+\pi / 2 n]\right| \\
& <\epsilon / 2+M O(1 / n)<\epsilon,
\end{aligned}
$$

where $M=\max _{-1 \leqq x \leqq+1}|f(x)|$, and this proves our theorem.

Budapest, Hungary

\title{
DISCONTINUOUS CONVEX SOLUTIONS OF DIFFERENCE EQUATIONS ${ }^{1}$
}

\section{FRITZ JOHN}

This paper contains some conditions for continuity of convex solutions of a difference equation.

A function $f(x)$ defined for $a \leqq x \leqq b$ is convex, if

$$
\left(\frac{x+y}{2}\right) \leqq \frac{f(x)+f(y)}{2} .
$$

If $f(x)$ is convex and bounded from above in $a \leqq x \leqq b$, then $f(x)$ is continuous (see Bernstein [1, p. 422]). ${ }^{2}$ If $f(x)$ is convex in $a \leqq x \leqq b$ and $y$ a fixed number with $a<y<b$, let the function $\phi_{y}(x)$ be defined by

$$
\phi_{y}(x)=\lim _{\alpha \rightarrow x-y} f(y+\alpha),
$$

where $\alpha$ assumes rational values only. Then $\phi_{y}(x)$ is uniquely defined, continuous, and convex for $a<x<b$ (F. Bernstein [1, p. 431, Theorem 7]); moreover $\phi_{y}(x)=f(x)$ for rational $y-x$.

THEOREM 1. If there exists at most one continuous convex solution of the difference equation

$$
F(x, f(x), f(x+1), \cdots, f(x+n))=g(x), \quad x>0,
$$

where $F$ and $g$ are continuous functions of their arguments, then there exist no discontinuous convex solutions.

Proof. If $f(x)$ is a convex solution, then, for $x-y$ rational,

$$
F\left(x, \phi_{y}(x), \phi_{y}(x+1), \cdots, \phi_{y}(x+n)\right)=g(x) ;
$$

\footnotetext{
1 Presented to the Society, September 12, 1940.

2 The numbers in brackets refer to the bibliography.
} 
as both members of this equation are continuous functions of $x$, it holds for all $x>0$. As there is at most one continuous convex solution, we have

$$
\phi_{y}(x)=\phi_{z}(x)
$$

for all positive $z, y, x$. As $\phi_{z}(z)=f(z)$, we see that $f(z)$ is identical with the continuous function $\phi_{y}(z)$ for all positive $z$.

TheOREM 2. If the difference equation (2) has at most one solution, which is monotone for sufficiently large $x$, then (2) has at most one convex solution, and that solution will be continuous.

PRoof. Every continuous convex solution is monotone for sufficiently large $x$. Apply Theorem 1 .

TheOREM 3. A difference equation of the form

$$
\prod_{k=0}^{n}(f(x+k))^{a_{k}}=g(x), \quad x>0,
$$

( $a_{k}$ real constants) has at most one convex solution, if

(1) all roots of the equation

$$
\sum_{k=0}^{n} a_{k} x^{k}=0
$$

are simple and of absolute value 1 ,

$$
a_{n}>0, \quad \sum_{k=0}^{n} a_{k} \neq 0,
$$

(2) $g(x) \neq 0$ and continuous,

(3) $\lim _{x \rightarrow \infty}(\log |g(x)|) / x=0$,

(4) $\lim _{x \rightarrow \infty}(\log |g(x)|) / \log x \neq \sum_{k} a_{k}$.

Proof. Assumption (3) implies

$$
\prod_{k=0}^{n}|f(x+k)|^{a_{k}}=|g(x)| .
$$

From assumption (1) above and the lemma proved below, it follows that there are non-negative constants $b_{l}$ and $c_{r}$, such that

$$
\begin{aligned}
\prod_{l}|g(x+l)|^{b_{l}} & =\prod_{l}\left[\prod_{k}|f(x+k+l)| a^{a_{k}}\right]^{b_{l}} \\
& =\prod_{r}|f(x+r)|^{c_{r}} .
\end{aligned}
$$


For every continuous convex solution $f(x)$ of $(3),|f(x)|$ is either monotone non-decreasing or monotone decreasing for sufficiently large $x$. Which of these alternatives takes place is determined by $g(x)$, as in the first case

$$
\prod_{l}|g(x+l)| b_{l}
$$

is monotone non-decreasing, and in the second case monotone decreasing for sufficiently large $x$. If $|f(x)|$ is monotone non-decreasing, we have in $F(x)=\log |f(x)|$ a non-decreasing solution of the equation

$$
\sum_{k} a_{k} F(x+k)=\log |g(x)| \text {. }
$$

It follows from our assumptions that such a solution $F(x)$ is uniquely determined for all sufficiently large $x$, and hence for all $x$ (John [3, p. 183]). If $|f(x)|$ is monotone decreasing for sufficiently large $x$, then $F(x)=-\log |f(x)|$ is an increasing solution of

$$
\sum_{k} a_{k} F(x+k)=-\log |g(x)|,
$$

and hence uniquely determined. Thus for any continuous convex solution $f(x)$ of (3), $|f(x)|$ is uniquely determined. Then $f(x)$ is uniquely determined as well, unless $f(x)$ is linear for sufficiently large $x$; but if $f(x)$ is linear for large $x$,

$$
\lim _{x \rightarrow \infty} \frac{\log |g(x)|}{\log x}=\lim _{x \rightarrow \infty} \sum_{k} a_{k} \frac{\log |f(x+k)|}{\log x}=\sum_{k} a_{k}
$$

contrary to assumption. Thus there exists at most one continuous convex solution, and hence at most one convex solution.

Example. The equation

$$
f(x+1) \cdot f(x)=x^{-p}, \quad x>0, p>0,
$$

satisfies the assumptions of Theorem 3 and hence has at most one convex solution (proved by A. E. Meyer [4] for $p=1$, for general $p$ by H. P. Thielman [5]). The convex solution is found to be

$$
B\left(\frac{1}{2} x, \frac{1}{2}\right)^{p} /(2 \pi)^{p / 2} \text {. }
$$

LemmA. If $\phi(x)=\sum_{k=0}^{n} a_{k} x^{k}$ is a polynomial, such that

(1) $a_{n}>0$,

(2) $\phi(x)$ has no positive real roots, then there are polynomials $\psi(x)$ and $\sigma(x)$ with non-negative coefficients, such that

$$
\phi(x) \cdot \psi(x)=\sigma(x) .
$$


Proof. $\phi(x)$ can be factored in the form

$$
\phi(x)=a_{n} \prod_{l}\left(x+\alpha_{l}\right) \prod_{k}\left(x^{2}+2 \beta_{k} x+\gamma_{k}\right)
$$

where $\alpha_{l} \geqq 0, \gamma_{k}>\beta_{k}^{2}$. Hence it is sufficient to prove the lemma for the case that

$$
\phi(x)=x^{2}+2 \beta x+\gamma
$$

and $\beta^{2}<\gamma$. We define $\theta$ with $0<\theta<\pi$ by

$$
\cos \theta=-\beta / \gamma^{1 / 2} \text {. }
$$

Let the non-negative integer $s$ be determined by

Put

$$
\frac{\pi}{s+2} \leqq \theta<\frac{\pi}{s+1} .
$$

$$
\begin{aligned}
\psi(x) & =\frac{\left(\gamma^{1 / 2}\right)^{s+2} \sin (s+1) \theta-x\left(\gamma^{1 / 2}\right)^{s+1} \sin (s+2) \theta+x^{s+2} \sin \theta}{\left[x^{2}+2 \beta x+\gamma\right] \cdot \sin \theta} \\
& =\sum_{k=0}^{s}\left(\gamma^{1 / 2}\right)^{k} \frac{\sin (k+1) \theta}{\sin \theta} x^{s-k} .
\end{aligned}
$$

$\psi(x)$ and $\left(x^{2}+2 \beta x+\gamma\right) \cdot \psi(x)$ obviously are polynomials with non-negative coefficients.

THEOREM 4. The difference equation

$$
\sum_{k=0}^{n} a_{k} f(x+k)=g(x), \quad x>0,
$$

has no discontinuous convex solutions, if

(a) $g(x)$ is bounded from above in every positive interval,

(b) $a_{n}>0$,

(c) the equation $\sum_{k=0}^{n} a_{k} x^{k}=0$ has no positive real roots.

Proof. Let $\phi(x)=\sum_{k=0}^{n} a_{k} x^{k}$. Let $\psi(x)$ be a polynomial, such that $\psi(x)$ and $\phi(x) \cdot \psi(x)$ have no negative coefficients. Let

$$
\phi(x) \cdot \psi(x)=x^{s} \cdot \sigma(x),
$$

where $\sigma(x)$ is a polynomial of degree $m$ with $\sigma(0) \neq 0$. Then

$$
\sigma(x) \cdot x^{m} \sigma\left(\frac{1}{x}\right)=\sum_{k=0}^{2 m} c_{k} x^{k}
$$


is a polynomial of degree $2 m$ with $c_{k}=c_{2 m-k} \geqq 0$. Put

Then

$$
\tau(x)=\psi(x) \cdot x^{m} \sigma\left(\frac{1}{x}\right)=\sum_{r} b_{r} x^{r} .
$$

$$
\phi(x) \cdot \tau(x)=x^{s} \sum_{k=0}^{2 m} c_{k} x^{k},
$$

where $\tau(x)$ is a polynomial with non-negative coefficients $b_{r}$ and $c_{k}=c_{2 m-k} \geqq 0$. For a convex solution $f(x)$ of $(4)$

$$
\begin{aligned}
\sum_{r} b_{r} g(x+r) & =\sum_{k=0}^{2 m} c_{k} f(x+k+s) \\
& =\frac{1}{2} \sum_{k=0}^{2 m} c_{k}[f(x+k+s)+f(x+2 m-k+s)] \\
& \geqq \frac{1}{2} \sum_{k=0}^{2 m} c_{k} f(x+s+m),
\end{aligned}
$$

or

$$
f(x+s+m) \leqq \frac{2 \sum_{r} b_{r} g(x+r)}{\sum_{k} c_{k}} .
$$

As $g(x)$ is bounded above, it follows that $f(x)$ is bounded above, and hence continuous.

THEOREM 5. If the difference equation

$$
\sum_{0=k}^{n} a_{k} f(x+k)=g(x)
$$

has a continuous convex solution, and if the equation $\sum_{k=0}^{n} a_{k} x^{k}=0$ has a positive real root, then the difference equation has discontinuous convex solutions as well.

PrOoF. It is sufficient to prove that the equation

$$
\sum_{k=0}^{n} a_{k} f(x+k)=0
$$

has a discontinuous convex solution, as the sum of two convex functions is again convex. Let

$$
\sum_{k=0}^{n} a_{k} x^{k}=(x-\lambda) \sum_{k=0}^{n-1} b_{k} x^{k}
$$


where $\lambda>0$. Then

$$
\sum_{k} a_{k} f(x+k)=\sum_{k} b_{k}[f(x+k+1)-\lambda f(x+k)] .
$$

It is sufficient to show that the equation

$$
f(x+1)-\lambda f(x)=0
$$

has a discontinuous convex solution.

Let $\Sigma$ be a basis for all real numbers; that is, every real $x$ may be represented in one and only one way in the form

$$
x=\alpha a+\beta b+\cdots+\gamma c,
$$

where $a, b, \cdots, c$ are in $\Sigma$, and $\alpha, \beta, \cdots, \gamma$ are rational numbers. (The existence of such a base is proved by Hamel [2].) Without restriction of generality we may assume 1 to be an element of $\Sigma$ (this comes back to assuming a normal ordering of the set of real numbers with 1 as first element). For every real $x$, there is then uniquely determined a number $\alpha$, such that

$$
x=\alpha \cdot 1+\beta b+\cdots+\gamma c,
$$

where $\alpha, \beta, \cdots, \gamma$ are rational, and $1, b, \cdots, c$ are in $\Sigma$. If $\lambda \neq 1$, define $f(x)$ by $\lambda^{\alpha}$. Then

$$
f(x+1)-\lambda f(x)=\lambda^{\alpha+1}-\lambda \cdot \lambda^{\alpha}=0 .
$$

If $y=\alpha^{\prime} 1+\beta^{\prime} b+\cdots+\gamma^{\prime} c$ is the representation of $y$ then

$$
f\left(\frac{x+y}{2}\right)=\left(\lambda^{\alpha} \cdot \lambda^{\alpha^{\prime}}\right)^{1 / 2} \leqq \frac{\lambda^{\alpha}+\lambda^{\alpha^{\prime}}}{2}=\frac{f(x)+f(y)}{2} .
$$

Hence $f(x)$ is a convex solution of (6). $f(x)$ is discontinuous in every interval, as for an element $b \neq 1$ of $\Sigma$

$$
f(\beta b)=1
$$

for all rational $\beta$, whereas

$$
f(\alpha)=\lambda^{\alpha} \neq 1
$$

for all rational $\alpha \neq 0$. Similarly, if $\lambda=1$, we define $f(x)=\beta$, where $\beta$ is the coefficient of the fixed element $b \neq 1$ of $\Sigma$. Then

$$
f(x+1)-f(x) \equiv 0, \quad f\left(\frac{x+y}{2}\right) \equiv \frac{f(x)+f(y)}{2},
$$

$f(\alpha)=0$ for all rational $\alpha$, but $f(\beta b)=\beta \neq 0$ for all rational $\beta \neq 0$. 
Note added March 15: I am indebted to Professors O. Szász and G. Szegö for the information that the Lemma had been proved by E. Meissner, Mathematische Annalen, vol. 70 (1911), pp. 223-235. See also Pólya and Szegö, Aufgaben und Lehrsätze aus der Analysis, vol. 2, p. 730, no. 190.

\section{BIBLIOGRAPHY}

1. F. Bernstein, Über das Gauss'sche Fehlergesetz, Mathematische Annalen, vol. 64 (1907), pp. 417-447.

2. G. Hamel, Eine Basis aller Zahlen und die unstetigen Lösungen der Funktionalgleichung $f(x+y)=f(x)+f(y)$, Mathematische Annalen, vol. 60 (1905), pp. 459-462.

3. F. John, Special solutions of certain difference equations, Acta Mathematica, vol. 71 (1939), pp. 175-189.

4. A. E. Meyer, Konvexe Lösung der Funktionalgleichung $1 / f(x+1)=x f(x)$, Acta Mathematica, vol. 70 (1939), pp. 57-62.

5. H. P. Thielman, On the convex solution of a certain functional equation, this Bulletin, vol. 47 (1941), pp. 118-120.

UNIVERSITY OF KeNTUCKY 